\title{
Multivisceral resection for gastric cancer: a systematic review
}

\author{
Savtaj S. Brar • Rajini Seevaratnam • \\ Roberta Cardoso - Lavanya Yohanathan • \\ Calvin Law $\cdot$ Lucy Helyer $\cdot$ Natalie G. Coburn
}

Received: 28 February 2011/ Accepted: 26 June 2011/Published online: 23 July 2011

(c) The International Gastric Cancer Association and The Japanese Gastric Cancer Association 2011

\begin{abstract}
Background The overall prognosis and survival of patients with advanced gastric cancer is generally poor. One of the most powerful predictors of outcomes in gastric cancer surgery is an R0 resection. However, the extent of the required surgical resection and the additional benefit of multivisceral resection (MVR) are controversial.

Methods Electronic literature searches were conducted using Medline, EMBASE, and the Cochrane Central Register of Controlled Trials from January 1, 1998 to December 31, 2009. All search titles and abstracts were independently rated for relevance by a minimum of two reviewers.

Results Seventeen studies were included in this review. Among the 1343 patients who underwent MVR, overall complication rates ranged from 11.8 to $90.5 \%$. Perioperative mortality was found to be $0-15 \%$. Pathological T4 disease was confirmed in $28.8-89 \%$ of patients. R0 resection and
\end{abstract}

Electronic supplementary material The online version of this article (doi:10.1007/s10120-011-0074-9) contains supplementary material, which is available to authorized users.

S. S. Brar · C. Law · N. G. Coburn $(\bowtie)$

Division of Surgical Oncology, Odette Cancer Centre,

Sunnybrook Health Sciences Centre, University of Toronto,

Suite T2-60, 2075 Bayview Ave, Toronto,

ON M4N 3M5, Canada

e-mail: natalie.coburn@sunnybrook.ca

S. S. Brar

The London School of Economics, London, UK

R. Seevaratnam $\cdot$ R. Cardoso $\cdot$ L. Yohanathan $\cdot$ N. G. Coburn Sunnybrook Research Institute, Sunnybrook Health Sciences Centre, Toronto, Canada

L. Helyer

Department of Surgery, Dalhousie University, Halifax, Canada extent of nodal involvement were important predictors of survival in patients undergoing MVR. Patient outcomes may also be affected by the number of organs resected.

Conclusions Gastrectomy with MVR can be safely pursued in patients with locally advanced gastric cancer to achieve an R0 resection. MVR may not be beneficial in patients with extensive nodal disease.

Keywords Gastric cancer $\cdot$ Surgery $\cdot$ Multivisceral resection $\cdot$ Margins

\section{Introduction}

For patients undergoing curative surgery for gastric cancer, $\mathrm{R} 0$ resection (negative microscopic and macroscopic margins) is the most powerful predictor of outcome [1]. In the West, unfortunately, gastric cancer typically presents at an advanced stage, and tumor invasion into adjacent structures (International Union Against Cancer [UICC]/American Joint Committee on Cancer [AJCC] Stage T4) is present in many of these patients [2,3]. Though patients with T4 gastric cancer will frequently present with peritoneal dissemination or distant metastases, many do not have M1 disease and thus are candidates for curative-intent surgery.

In patients with $\mathrm{T} 4$ gastric cancer, a multivisceral resection (MVR), or gastrectomy with resection of adjacent organs, is required to achieve an R0 resection. Observations of increased perioperative morbidity and mortality with MVR are widespread [4-6]. The increased operative risk of MVR must be weighed against the evidence that only selected patients may benefit from the radical operation. As a result, there exists a lack of consensus regarding its use in the treatment of locally advanced gastric cancer. As true histological invasion into adjacent organs is difficult to identify 
with pre-operative imaging or even during an operation, the decision to proceed with MVR is challenging [2, 7].

This review will identify and synthesize the findings of studies on surgical and long-term outcomes in patients undergoing MVR for gastric cancer involving adjacent organs.

\section{Methods}

Data sources

Electronic literature searches were conducted in Medline and EMBASE from January 1, 1998 to December 31, 2009

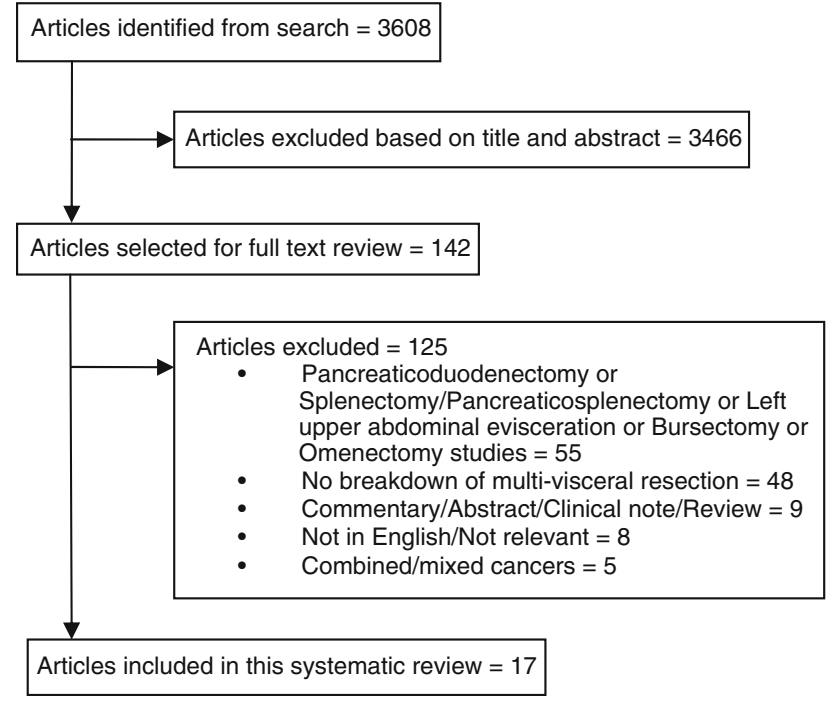

Fig. 1 Article selection flow according to the search algorithm presented in Appendix A. Search terms included [exp Stomach Cancer/or (((gastric or stomach) adj1 cancer\$) or ((gastric or stomach) adj1 carcinoma) or ((gastric or stomach) adj1 adenocarcinoma) or ((gastric or stomach) adj1 neoplasm\$)).mp.] and [((negative or resection) adj2 margin\$).mp. or exp frozen section/or exp GASTRECTOMY/or ((gastric or stomach) adj2 resect\$).mp. [mp=title, abstract, subject headings, heading word, drug trade name, original title, device manufacturer, drug manufacturer name] or omentectom\$.mp. or multivisceral resection\$.mp.] and [clinical trial/or controlled clinical trial/or exp comparative study/or meta-analysis/or multicenter study/or exp practice guideline/or randomized controlled trial/] not [Case Report/or review]. A separate search of the Cochrane Central Register of Controlled Trials (1998-2009) was performed using the search term gastric cancer. No attempt was made to locate unpublished material.

Study selection and review process

To be eligible, studies had to meet the following criteria: (1) investigation of MVR in newly (not recurrent) diagnosed patients with histopathology-confirmed gastric adenocarcinoma; (2) patients underwent surgery, and complication or survival data was reported; (3) involved human patients with a minimum of 30 patients; (4) published in peer-reviewed journals from 1998 to 2009; and (5) published in English. Studies were excluded according to the following exclusion criteria: (1) involved animals and/or ex vivo samples; (2) involved patients with mixed cancer or studies investigating MVR in other cancers with no separate analysis of gastric cancer subjects; (3) studies

Table 1 Organs resected during MVR

\begin{tabular}{|c|c|c|c|c|c|c|c|c|}
\hline \multirow[t]{2}{*}{ Study } & \multirow[t]{2}{*}{ Patients $(N)$} & \multicolumn{7}{|c|}{ Organs resected } \\
\hline & & $\mathrm{Sp}$ & $\mathrm{P}$ & LB & SB & $\mathrm{L}$ & GB & $\mathrm{O}$ \\
\hline Carboni [2] & 65 & 31 & 28 & 16 & 1 & 12 & 2 & 10 \\
\hline Colen [8] & 21 & 13 & 12 & 5 & 3 & 2 & - & - \\
\hline D'Amato [9] & 52 & - & 32 & 6 & 1 & 4 & - & 5 \\
\hline Jeong [11] & 60 & 33 & 43 & 17 & 4 & 5 & 4 & 6 \\
\hline Kim [12] & 34 & 13 & 10 & 19 & - & 4 & 1 & - \\
\hline Martin [1] & 418 & 251 & 65 & 36 & 27 & 33 & 27 & 537 \\
\hline Oñate-Ocaña [15] & 76 & 50 & 19 & 9 & 1 & 7 & - & 13 \\
\hline Otsuji [16] & 27 & - & 15 & 10 & - & 2 & - & - \\
\hline Ozer [17] & 56 & - & 30 & 18 & 2 & 10 & 2 & 5 \\
\hline Persiani [6] & 51 & 43 & 13 & 5 & - & 4 & - & 3 \\
\hline Shchepotin [19] & 353 & 150 & 243 & 159 & - & 101 & - & - \\
\hline Yong [20] & 20 & 11 & 10 & 10 & - & 3 & - & - \\
\hline
\end{tabular}

$M V R$ multivisceral resection, $S p$ spleen, $P$ pancreas, $L B$ large bowel, $S B$ small bowel, $L$ liver, $G B$ gallbladder, $O$ other (includes kidney, adrenal gland, uterus, ovary, mesentery, diaphragm, lung, pericardium) 
that did not provide sufficient information to determine complication or survival data; (4) review articles, metaanalyses, abstracts, conference proceedings, editorials/ letters, and case reports; and (5) studies investigating pancreaticoduodenectomy. An a priori decision to review pancreaticoduodenectomy in a separate study was made due to the operative risks unique to this procedure. All electronic search titles, selected abstracts, and full-text articles were independently reviewed by a minimum of two reviewers (NC, LH, RC, RS). Reference lists from review papers and relevant articles were also examined for additional studies that met our inclusion criteria. Disagreements on study inclusion/exclusion were resolved with a consensus meeting.

\section{Data extraction}

A systematic approach to data extraction was used to produce a descriptive summary of participants, interventions, and study findings. Two reviewers (RS, LY) independently extracted the data and a third reviewer (SB) checked the data extraction. No attempt was made to contact authors for additional information.

\section{Results}

\section{Literature search}

A total of 3608 titles/abstracts were identified from the electronic searches and reference lists for preliminary review. After removal of duplicates and screening for relevant titles and abstracts, a total of 142 articles were submitted for a full-text review. Seventeen studies involving 1343 patients met our inclusion criteria and were included in this review [1, 2, 4-6, 8-20] (Fig. 1).

\section{Morbidity and mortality}

Table 1 reports the organs resected along with gastrectomy for patients undergoing MVR. The most commonly resected organs were spleen, distal pancreas, liver, and large bowel (mostly transverse colon). Other commonly resected organs included small bowel and gallbladder. Less commonly resected organs included kidney, adrenal gland, mesocolon, diaphragm, lung, pericardium, ovary, and uterus. Reported complications from the studies examining MVR are listed in Table 2. Among the 734 patients with reported complications following MVR, 3\% had anastomotic leaks, $2 \%$ had pancreatic fistulas, and $10 \%$ had reported perioperative death, with overall complications rates ranging from 11.8 to $90.5 \%$. Perioperative mortality ranged from 0 to $15 \%$.

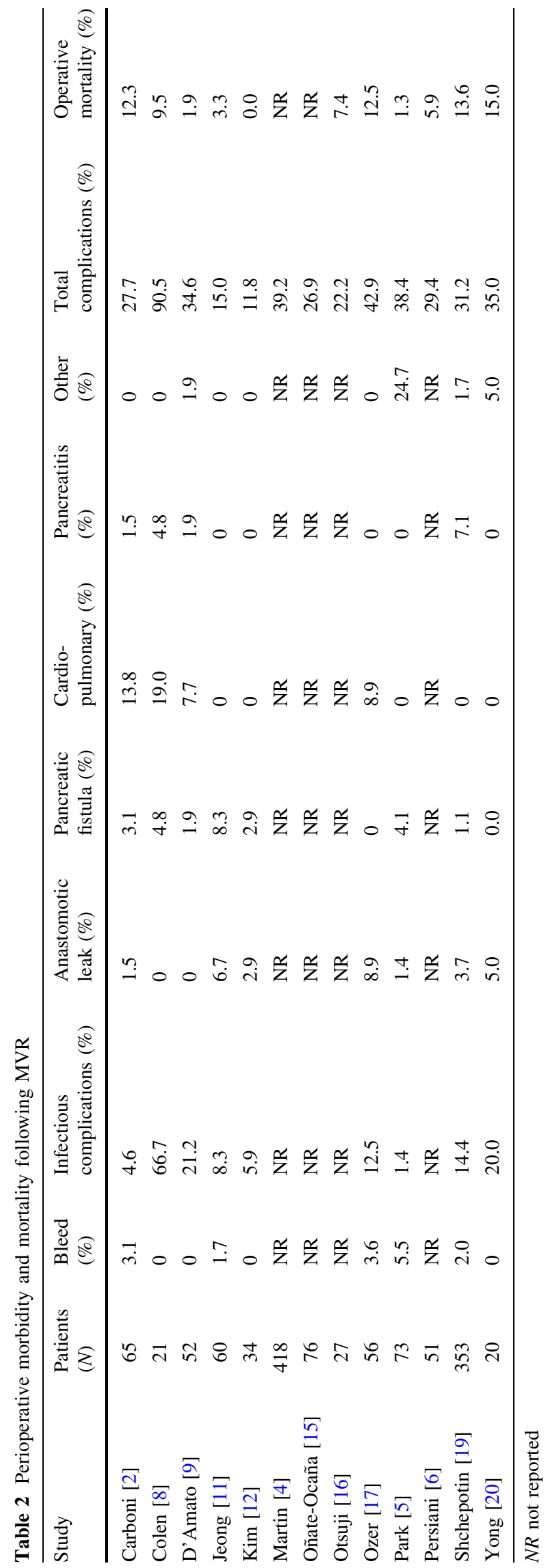


Table 3 Overall survival following MVR
Significance corresponds to the noted superscripts

$N A$ not applicable, $N R$ not reported, $N S$ not significant, $M S$ median survival

\begin{tabular}{|c|c|c|c|c|}
\hline Study & Patients $(N)$ & Pathologic T4 (\%) & Overall survival (\%) & Significance \\
\hline Carboni [2] & $\mathrm{MVR}=65$ & 80.0 & 5 year: 21.8 & NA \\
\hline Colen [8] & MVR $=21$ & 38.1 & 5 year: 30 & NA \\
\hline D'Amato [9] & $\mathrm{MVR}=52$ & 28.8 & $\begin{array}{l}3 \text { year: } 41 \\
5 \text { year: } 31\end{array}$ & NA \\
\hline Isozaki [10] & $\mathrm{MVR}=86$ & NR & 5 year: 35 & NA \\
\hline \multirow[t]{2}{*}{ Jeong [11] } & $\mathrm{MVR}=60$ & 63.4 & $\begin{array}{l}1 \text { year: } 73.1 \\
2 \text { year: } 49.7 \\
3 \text { year: } 36.4\end{array}$ & NS \\
\hline & No MVR = 11 & & NR & \\
\hline Kim [12] & $\begin{array}{l}\text { MVR }=34 \\
\text { No } M V R=45\end{array}$ & NR & $\begin{array}{l}5 \text { year: } 37.8 \\
5 \text { year: } 29.4\end{array}$ & NS \\
\hline Kitamura [13] & MVR $=174$ & NR & 5 year: 15 & NA \\
\hline \multirow[t]{2}{*}{ Lee [14] } & $\mathrm{MVR}=21$ & NR & $\begin{array}{l}\text { MS: } 15 \text { months }{ }^{\mathrm{a}} \\
5 \text { year: } 10^{\mathrm{a}}\end{array}$ & $P<0.05^{\mathrm{a}}$ \\
\hline & No MVR = 169 & & $\begin{array}{l}\text { MS: } 23 \text { months }^{\mathrm{a}} \\
5 \text { year: } 25^{\mathrm{a}}\end{array}$ & \\
\hline \multirow[t]{2}{*}{ Martin [4] } & $\mathrm{MVR}=268$ & 13.8 & $\begin{array}{l}\text { MS: } 32 \text { months }{ }^{\mathrm{b}} \\
5 \text { year: } 40\end{array}$ & $P=0.001^{\mathrm{b}}$ \\
\hline & No MVR $=865$ & NR & $\begin{array}{l}\text { MS: } 63 \text { months }{ }^{\text {b }} \\
5 \text { year: } 52\end{array}$ & \\
\hline Oñate-Ocaña [15] & $\mathrm{MVR}=74$ & NR & MS: 30.5 months & NA \\
\hline Otsuji [16] & $\begin{array}{l}\text { MVR }=27 \\
\text { No } M V R=28\end{array}$ & NR & $\begin{array}{l}5 \text { year: } 22.7 \\
5 \text { year: } 5.9\end{array}$ & NS \\
\hline Ozer [17] & $\mathrm{MVR}=56$ & 66.1 & $\begin{array}{l}\text { MS: } 13.3 \text { months } \\
1 \text { year: } 53.3 \\
2 \text { year: } 36 \\
3 \text { year: } 28.1\end{array}$ & NA \\
\hline Saito [18] & No MVR = 74 & NR & $\begin{array}{l}\text { R0, } 1 \text { year: } 65^{\mathrm{c}, \mathrm{d}} \\
\text { R0, } 5 \text { year: } 35^{\mathrm{c}, \mathrm{d}} \\
\mathrm{R}+, 1 \text { year: } 35^{\mathrm{c}} \\
\mathrm{R}+, 5 \text { year: } 0^{\mathrm{c}} \\
1 \text { year: } 25^{\mathrm{d}} \\
5 \text { year: } 0^{\mathrm{d}}\end{array}$ & $P<0.001^{\mathrm{c}, \mathrm{d}}$ \\
\hline Shchepotin [19] & $\mathrm{MVR}=353$ & 89.0 & 5 year: 25 & NA \\
\hline Yong [20] & $\mathrm{MVR}=20$ & NR & $\begin{array}{l}\text { MS: } 17 \text { months } \\
1 \text { year: } 60 \\
2 \text { year: } 30 \\
3 \text { year: } 0\end{array}$ & NA \\
\hline
\end{tabular}

\section{Survival}

Table 3 reports survival data from MVR studies. Longterm survival was reported in studies as median survival in months or as percentage survival at 1,2,3, or 5 years. Five of fifteen studies reported median survival (range 13-32 months) $[4,14,15,17,20]$. Four of fifteen studies reported 3-year survival (range $0-41 \%$ ) $[9,11,17,20]$. Eleven of fifteen studies reported 5-year survival (range $0-40 \%)[2,4,8-10,12-14,16,18,19]$. One study reported a statistically significant survival advantage when MVR was performed for patients with locally invasive disease when compared to gastrectomy alone [2]. However, two studies reported a statistically significant survival advantage for gastrectomy alone compared to MVR [4, 14].

Table 4 lists the studies that reported completion of resection. An R0 resection was performed in $58-100 \%$ of patients. Four studies reported a statistically significant survival advantage when MVR was performed with an R0 resection compared to $R+$ resection $[2,9,11,18]$. Table 5 
Table 4 Survival following MVR by completion of resection

\begin{tabular}{|c|c|c|c|c|}
\hline Study & Patients $(N)$ & $\begin{array}{l}\mathrm{R} 0 \text { versus } \mathrm{R}+ \\
\text { (\% of patients) }\end{array}$ & $\begin{array}{l}\text { Survival by R0 } \\
\text { status }\end{array}$ & Significance \\
\hline Carboni [2] & $\mathrm{MVR}=65$ & $\begin{array}{l}\text { R0: } 61.5 \% \\
\text { R1: } 27.7 \% \\
\text { R2: } 10.8 \%\end{array}$ & $\begin{array}{l}\text { R0, } 5 \text { year: } 30.6 \%^{\mathrm{a}} \\
\text { R1, } 5 \text { year: } 0 \%^{\mathrm{a}}\end{array}$ & $P=0.001^{\mathrm{a}}$ \\
\hline D’Amato [9] & $\mathrm{MVR}=52$ & R0: $69 \%$ & $\begin{array}{l}\mathrm{R} 0,5 \text { year: } 45 \%^{\mathrm{b}} \\
\mathrm{R}+, 5 \text { year: } 0 \%^{\mathrm{b}}\end{array}$ & $P<0.001^{\mathrm{b}}$ \\
\hline Jeong [11] & $\mathrm{MVR}=60$ & $\begin{array}{l}\mathrm{R} 0: 78.3 \% \\
\mathrm{R}+: 21.7 \%\end{array}$ & $\begin{array}{l}\text { R0, } 1 \text { year: } 74 \%^{\mathrm{c}} \\
\text { R0, } 2 \text { year: } 56.5 \%^{\mathrm{c}} \\
\text { R0, } 3 \text { year: } 47.5 \%^{\mathrm{c}} \\
\text { R+, } 1 \text { year: } 57.1 \%^{\mathrm{c}} \\
\text { R+, } 2 \text { year: } 26.7 \%^{\mathrm{c}}\end{array}$ & $P=0.046^{\mathrm{c}}$ \\
\hline & No $M V R=11$ & $\mathrm{R}+: 100 \%$ & $\begin{array}{l}\mathrm{R}+, 1 \text { year: } 68.5 \% \\
\mathrm{R}+, 2 \text { year: } 24 \%\end{array}$ & \\
\hline $\operatorname{Kim}[12]$ & $\begin{array}{l}\text { MVR }=34 \\
\text { No } M V R=45\end{array}$ & $\begin{array}{l}\text { R0: } 76.5 \% \mathrm{R}+: 23.5 \% \\
\text { R0: } 37.8 \% \mathrm{R}+: 62.2 \%\end{array}$ & $\mathrm{NR}$ & NA \\
\hline Martin [4] & $\begin{array}{l}\text { MVR }=268 \\
\text { No } M V R=865\end{array}$ & $\begin{array}{l}\text { R0: } 100 \% \\
\text { R0: } 100 \%\end{array}$ & NR & NA \\
\hline Oñate-Ocaña [15] & $\mathrm{MVR}=74$ & $\begin{array}{l}\text { R0: } 58.1 \% \\
\text { R1: } 18.9 \% \\
\text { R2: } 23 \%\end{array}$ & NR & NA \\
\hline Saito [18] & $\mathrm{MVR}=82$ & $\begin{array}{l}\text { R0: } 67.1 \% \\
\mathrm{R}+: 32.9 \%\end{array}$ & $\begin{array}{l}\text { R0, } 1 \text { year: } 65 \%^{\mathrm{d}} \\
\text { R0, } 5 \text { year: } 35 \%^{\mathrm{d}} \\
\text { R+, } 1 \text { year: } 35 \%^{\mathrm{d}} \\
\text { R+, } 5 \text { year: } 0 \%^{\mathrm{d}}\end{array}$ & $P<0.001^{\mathrm{d}}$ \\
\hline
\end{tabular}

Significance corresponds to the noted superscripts

$N A$ not applicable, $N R$ not reported from 1.9 to $15.0 \%$, with five-year survival rates of $0-40 \%$. These studies have attempted to identify predictors of longterm survival, including margin positivity, the number of organs resected, UICC/AJCC stage, and other factors, which may aid in appropriate patient selection for MVR.

MVR is performed to provide patients with advanced gastric cancer the best chance at survival with a curative R0 resection. Eight studies reported their ability to achieve a complete resection (R0), with results ranging from 38 to $100 \%$, suggesting that a significant proportion of patients have positive microscopic or macroscopic margins despite undergoing MVR [1, 2, 7-9, 11, 17, 19]. Survival in patients who underwent MVR without a complete resection was demonstrated to be significantly diminished compared to those who had an R0 resection [2, 9, 11, 18]. Poor outcomes appear to be associated with both microscopic and macroscopic positive margins $[12,15]$. There were no 5 -year survivors in three studies that reported outcomes in patients undergoing MVR with residual tumor; however, for patients in whom an R0 resection was achieved, the 5 -year survival was $11.1-45.0 \%$ [2, 9, 18]. The importance of an R0 resection is highlighted by Saito and colleagues, who found patients who underwent MVR and had positive microscopic or macroscopic margins had nearly the same 
Table 5 Survival following MVR by organs involved or resected

\begin{tabular}{|c|c|c|c|}
\hline Study & $\begin{array}{l}\text { Patients } \\
(N)\end{array}$ & $\begin{array}{l}\text { Survival by } \\
\text { number of organs } \\
\text { involved or resected }\end{array}$ & Significance \\
\hline $\begin{array}{c}\text { Isozaki } \\
{[10]}\end{array}$ & $\mathrm{MVR}=86$ & $\begin{array}{l}1 \text { OI, } 5 \text { year: } 38.6 \%^{\mathrm{a}} \\
\geq 2 \text { OI, } 5 \text { year: } 10 \%^{\mathrm{a}}\end{array}$ & $P=0.0043^{\mathrm{a}}$ \\
\hline Martin [4] & MVR $=268$ & $\begin{array}{l}1 \text { OR, } 5 \text { year: } 42 \% \\
1 \text { OR, MS }=41 \text { months } \\
2 \text { OR, } 5 \text { year: } 32 \% \\
2 \text { OR, MS }=19 \text { months } \\
3 \text { OR, } 5 \text { year: } 28 \% \\
3 \text { OR, MS }=26 \text { months }\end{array}$ & NS \\
\hline Ozer [17] & $\mathrm{MVR}=56$ & $\begin{array}{l}1 \text { OR, } 1 \text { year: } 62.3 \%^{\mathrm{b}} \\
1 \text { OR, } 3 \text { year: } 40.8 \%^{\mathrm{b}} \\
\geq 2 \text { OR, } 1 \text { year: } 30 \%^{\mathrm{b}} \\
\geq 2 \text { OR, } 3 \text { year: } 6.4 \%^{\mathrm{b}}\end{array}$ & $P=0.003^{\mathrm{b}}$ \\
\hline Saito [18] & MVR $=82$ & $\begin{array}{l}1 \text { OI, R0, } 1 \text { year: } 75 \%^{\mathrm{c}, \mathrm{d}} \\
1 \text { OI, R0, } 5 \text { year: } 4 \%^{\mathrm{c}, \mathrm{d}} \\
1 \text { OI, R+, } 1 \text { year: } 28 \%^{\mathrm{c}} \\
1 \text { OI, R+, } 5 \text { year: } 0 \%^{\mathrm{c}} \\
\geq 2 \text { OI, R0, } 1 \text { year: } \\
70 \%{ }^{\mathrm{e}, \mathrm{f}} \\
\geq 2 \text { OI, R0, } 5 \text { year: } \\
35 \%{ }^{\mathrm{e}, \mathrm{f}} \\
\geq 2 \text { OI, R+, } 1 \text { year: } \\
30 \%^{\mathrm{e}} \\
\geq 2 \text { OI, R+, } 5 \text { year: } \\
0 \%^{\mathrm{e}}\end{array}$ & $\begin{array}{l}P<0.01^{\mathrm{c}} \\
P<0.001^{\mathrm{d}} \\
P<0.01^{\mathrm{e}} \\
P<0.05^{\mathrm{f}}\end{array}$ \\
\hline & $\begin{array}{l}\text { No } \\
\qquad \mathrm{MVR}=74\end{array}$ & $\begin{array}{l}1 \text { OI, } 1 \text { year: } 55 \%^{\mathrm{d}} \\
1 \text { OI, } 5 \text { year: } 0 \%^{\mathrm{d}} \\
\geq 2 \text { OI, } 1 \text { year: } 25 \%^{\mathrm{f}} \\
\geq 2 \text { OI, } 5 \text { year: } 0 \%^{\mathrm{f}}\end{array}$ & \\
\hline
\end{tabular}

Significance corresponds to the noted superscripts

$N A$ not applicable, $N S$ not significant, $M S$ median survival, $O I$ organs involved, $O R$ organs resected

outcomes as patients who did not undergo MVR [18]. Two studies reported decreased overall survival with MVR compared to gastrectomy alone [4, 14]. Comparisons in these studies, however, do not address the question of whether MVR is appropriate for patients with T4 gastric cancer. These two studies did not report the pathologic T4 status in the patients treated with gastrectomy alone [4, 14]. Further, Martin et al. [4] reported only $13.8 \%$ of patients who underwent MVR had pathologic T4 disease. These studies fail to address that the true comparator to MVR for patients with suspected $\mathrm{T} 4$ disease is gastrectomy alone with residual microscopic or macroscopic disease. Martin et al. [4] reported that $100 \%$ of patients in the gastrectomyalone group had an R0 resection, and therefore an MVR would not be indicated in these patients. Lee et al. [14] do not report R0 status data in their study for either the gastrectomy-alone or MVR groups. There is no evidence that gastrectomy alone, when yielding an R1 or R2 margin, is appropriate when R0 resection with MVR is safe and feasible. Studies that examined survival related to which organs were resected were few in number. However, resections involving the liver $[9,18]$ and the transverse colon [9] were found to be associated with increased survival compared to MVR with resection of other adjacent structures.

The goal of R0 resection must be balanced against the challenges in identifying whether gastric cancer is truly invading adjacent organs. Given the risk of morbidity, MVR ideally should be reserved for T4 lesions, with true histological invasion into adjacent organs [8]. Adhesions secondary to desmoplastic reaction can be mistaken for local invasion, especially when involving the pancreas [2]. Preoperative distinction between T3 and T4 lesions may be facilitated by preoperative imaging, including computed tomography (CT) and endoscopic ultrasound (EUS), although none of these factors appears to have high accuracy [22]. In this set of patients undergoing MVR with the goal of R0 resection, the percentage of patients with confirmed pathologic T4 disease ranged from 28.9 to $89 \%$ (Table 3). Difficulties in distinguishing adhesions from disease invasion will result in some patients undergoing a radical operation with increased likelihood of complication for no oncological benefit. The range between studies suggests there might be factors that may help identify patients with pathologic $\mathrm{T} 4$ disease, although none of these factors appears to have high accuracy. Identification and stratification with these factors must be balanced against the risk of leaving microscopic residual disease after resection, which, as discussed above, is associated with poor prognosis.

MVR may require resection of two or more organs along with gastrectomy in pursuit of negative margins. Four studies examined the number of organs involved or resected as a predictor of survival (Table 5) [4, 10, 17, 18]. Ozer et al. [17] found that patients who underwent MVR with 2 or more organs had a higher surgical morbidity, when adjusted for age, comorbidities, and stage, providing a possible explanation for the decreased survival for this group. As well, Martin et al. [1,4] report an increase in surgical complications with a trend towards lower 5-year survival when comparing one-, two-, and three-organ resections in addition to gastrectomy. In none of the studies are operative details such as estimated blood loss given, nor is it clear whether differences in survival in patients with 2 or more organs involved are a result of operative mortality or a marker of aggressive biology. However, Saito et al. [18] found that when an R0 resection was performed, there was no difference in survival when more than one organ was involved. Further, Saito et al. [18] found that when more than two organs adjacent to the stomach were involved, the patients who underwent an R0 
Table 6 Survival following MVR by tumor depth, nodal status, and stage

\begin{tabular}{|c|c|c|c|c|c|}
\hline Study & Patients $(N)$ & Survival by $\mathrm{T}$ status & Survival by $\mathrm{N}$ status & Survival by stage & Significance \\
\hline Carboni [2] & $\mathrm{MVR}=65$ & $\begin{array}{l}\mathrm{T} 3,5 \text { year: } 34.1 \%^{\mathrm{a}} \\
\mathrm{T} 4,5 \text { year: } 17.9 \%^{\mathrm{a}}\end{array}$ & $\begin{array}{l}\text { N0, } 5 \text { year: } 42.9 \%^{b} \\
N+, 5 \text { year: } 17.3 \%^{b}\end{array}$ & NR & $\begin{array}{l}P=0.07^{\mathrm{a}} \\
P=0.08^{\mathrm{b}}\end{array}$ \\
\hline D’Amato [9] & $\mathrm{MVR}=52$ & $\mathrm{NR}$ & $\begin{array}{l}\text { N0, } 5 \text { year: } 60 \%^{\mathrm{c}} \\
\text { N1, } 5 \text { year: } 40 \%^{\mathrm{c}} \\
\mathrm{N} 2,5 \text { year: } 0 \%^{\mathrm{c}} \\
\text { T4N+, } 5 \text { year: } 10 \%^{\mathrm{d}} \\
\text { T4N0, } 5 \text { year: } 42 \%^{\mathrm{d}}\end{array}$ & NR & $\begin{array}{l}P<0.01^{\mathrm{c}} \\
P<0.01^{\mathrm{d}}\end{array}$ \\
\hline Isozaki [10] & $\mathrm{MVR}=86$ & $\begin{array}{l}\text { T2, } 5 \text { year: } 46.7 \%^{\mathrm{e}} \\
\text { T3, } 5 \text { year: } 35.8 \% \\
\text { T4, } 5 \text { year: } 32.6 \%\end{array}$ & $\begin{array}{l}\text { N0, } 5 \text { year: } 74 \%^{\mathrm{f}, \mathrm{g}, \mathrm{h}} \\
\text { N1, } 5 \text { year: } 41.9 \%^{\mathrm{f}} \\
\text { N2, } 5 \text { year: } 8.4 \%^{\mathrm{g}} \\
\text { N3, } 5 \text { year: } 18.5 \%^{\mathrm{h}}\end{array}$ & NR & $\begin{array}{l}P=0.055^{\mathrm{e}} \\
P=0.059^{\mathrm{f}} \\
P=0.008^{\mathrm{g}} \\
P=0.014^{\mathrm{h}}\end{array}$ \\
\hline Jeong [11] & $\mathrm{MVR}=60$ & $\begin{array}{l}\text { T2-3, MS: } 30.2 \text { months } \\
\text { T4 MS: } 26.9 \text { months }\end{array}$ & $\begin{array}{l}\text { N0-N2, MS: } 31.2 \text { months }^{\mathrm{i}} \\
\text { N3 MS: } 13.3 \text { months }^{\mathrm{i}}\end{array}$ & NR & $P=0.001^{\mathrm{i}}$ \\
\hline Martin [4] & No MVR $=865$ & & & $\begin{array}{l}\text { Stage IIIA: } 25 \% \\
\text { Stage IIIB: } 15 \%^{\mathrm{j}} \\
\text { Stage IV: } 10 \% \\
\text { Stage IIIA: } 35 \% \\
\text { Stage IIIB: } 25 \%^{\mathrm{j}} \\
\text { Stage IV: } 22 \%\end{array}$ & $P=0.055^{\mathrm{j}}$ \\
\hline Ozer [17] & $\mathrm{MVR}=56$ & $\begin{array}{l}\text { T3, } 1 \text { year: } 58.7 \% \\
\text { T3, } 3 \text { year: } 51.3 \% \\
\text { T4, } 1 \text { year: } 51.9 \% \text { T4, } \\
\text { 3 year: } 18.8 \%\end{array}$ & $\begin{array}{l}\text { N0, } 1 \text { year: } 90 \%^{\mathrm{k}} \\
\text { N0, } 3 \text { year: } 77.1 \%^{\mathrm{k}} \\
\mathrm{N}+, 1 \text { year: } 41.4 \%^{\mathrm{k}} \\
\mathrm{N}+, 3 \text { year: } 17.1 \%^{\mathrm{k}}\end{array}$ & $\begin{array}{l}\text { Stage III, } 1 \text { year: } 74.9 \% \\
\text { Stage III, } 3 \text { year: } 53.9 \% \\
\text { Stage IV, } 1 \text { year: } 39.9 \% \\
\text { Stage IV, } 3 \text { year: } 12.3 \%\end{array}$ & $P<0.001^{\mathrm{k}}$ \\
\hline Saito [18] & $\mathrm{MVR}=82$ & NA & $\begin{array}{l}\text { N0-2, R0, } 1 \text { year: } 70 \%^{1, \mathrm{~m}} \\
\text { N0-2, R0, } 5 \text { year: } 50 \%^{1, \mathrm{~m}} \\
\text { N0-2, R+, } 1 \text { year: } 55 \%^{1} \\
\text { N0-2, R+, } 5 \text { year: } 0 \%^{1} \\
\text { N3-4, R0, } 1 \text { year: } 85 \%^{\mathrm{n}, \mathrm{o}} \\
\text { N3-4, R0, } 5 \text { year: } 11 \%^{\mathrm{n}, \mathrm{o}} \\
\text { N3-4, R+, } 1 \text { year: } 25 \%^{\mathrm{n}} \\
\text { N3-4, R+, } 5 \text { year: } 0 \%^{\mathrm{n}}\end{array}$ & $\mathrm{NR}$ & $\begin{array}{l}P<0.05^{1} \\
P<0.001^{\mathrm{m}} \\
P<0.05^{\mathrm{n}} \\
P<0.001^{\mathrm{o}}\end{array}$ \\
\hline & No $M V R=74$ & NA & $\begin{array}{l}\text { N0-2, } 1 \text { year: } 45 \%^{\mathrm{m}} \\
\text { N0-2, } 5 \text { year: } 0 \%^{\mathrm{m}} \\
\mathrm{N} 3-4,1 \text { year: } 12 \%^{\circ} \\
\mathrm{N} 3-4,5 \text { year: } 0 \%^{\circ}\end{array}$ & NR & \\
\hline Shchepotin [19] & $\mathrm{MVR}=353$ & NA & $\begin{array}{l}\text { N0, } 5 \text { year: } 37 \% \\
\mathrm{~N}+, 5 \text { year: } 15 \%\end{array}$ & NR & NR \\
\hline
\end{tabular}

Significance corresponds to the noted superscripts

Staging is based on AJCC 6th edition

$N A$ not applicable, $N R$ not reported, $M S$ median survival

resection had improved survival compared to those who did not undergo MVR. If resection of two or more organs is necessary for achieving negative margins, MVR should be pursued despite possible operative risks.

Overall survival is a complex issue as many factors influence a patient's outcome. Table 6 lists studies reporting survival data by tumor depth, extent of nodal involvement, and stage. Nodal status is highly predictive of survival in gastric cancer patients. Not surprisingly, several papers show survival after an MVR to be dependent on the nodal status of patients [2, 9-11, 17]. Jeong et al. [11] reported that patients with $\mathrm{N} 3$ disease showed no improved survival in the MVR group versus the group treated with gastrectomy alone, thus suggesting that the benefit of MVR 
to achieve an R0 resection may be limited by the burden of nodal involvement. Some authors conclude that extended MVR should not be completed when macroscopic nodal disease is present [13]. Nevertheless, MVR may benefit some patients even with extensive nodal involvement. Saito et al. [18] showed a statistically significant difference in 5-year survival between patients with N3 disease who underwent an MVR with R0 resection compared to patients with positive pathological margins or patients who did not undergo MVR. Thus, MVR may be suitable for appropriately selected patients, given the ability to achieve negative histological margins, even with extensive nodal involvement. From a practical standpoint, identifying preoperatively the patients with lymph node metastasis may be difficult, given the limited accuracies of currently available diagnostic imaging to detect macroscopic nodal disease [11].

Conclusions drawn from this review must consider the many limitations of the included studies. Most are retrospective case series from single institutions, and, furthermore, many are based in Asian countries, possibly limiting the applicability of results to Western centers. One limitation of our review is that exclusion of patients for whom MVR was performed for reasons other than en-bloc resection may not have occurred in all studies [8]. Additionally, in this review, we have excluded patients who underwent pancreaticoduodenectomy, given the technical complexity of the operation, and the specific considerations of this procedure will be discussed in a separate review.

\section{Conclusion}

Gastrectomy with MVR can be pursued in patients with locally advanced gastric cancer with the goal of R0 resection. Morbidity and mortality may be increased, but the benefit of attaining an $\mathrm{R} 0$ resection has a positive impact on overall patient survival. Patient selection for MVR must take into account nodal status and the number of organs involved, and care must be taken to attempt to identify true histological invasion before and during resection.

Acknowledgments This research is funded by the Canadian Cancer Society (grant \#019325). Dr. Coburn has received salary support for this work through the Ontario Ministry of Health and Long-Term Care Career Scientist Award. Dr. Law is supported by the Hanna Family Chair in Surgical Oncology.

\section{References}

1. Martin R, Jaques D, Brennan M, Karpeh M. Achieving R0 resection for locally advanced gastric cancer: is it worth the risk of multiorgan resection? J Am Coll Surg. 2002;194(5):568-77.
2. Carboni F, Lepiane P, Santoro R, Lorusso R, Mancini P, Sperduti $\mathrm{I}$, et al. Extended multiorgan resection for T4 gastric carcinoma: 25 year experience. J Surg Oncol. 2005;90(2):95-100.

3. Edge S, Byrd D, Compton C, Fritz A, Greene F, Trotti A. American Joint Committee on Cancer cancer staging manual. New York: Springer; 2009.

4. Martin R, Jaques D, Brennan M, Karpeh M. Extended local resection for advanced gastric cancer: increased survival versus increased morbidity. Ann Surg. 2002;236(2):159.

5. Park D, Lee H, Kim H, Yang H, Lee K, Choe K. Predictors of operative morbidity and mortality in gastric cancer surgery. Br J Surg. 2005;92(9):1099-102.

6. Persiani R, Antonacci V, Biondi A, Rausei S, La Greca A, Zoccali M, et al. Determinants of surgical morbidity in gastric cancer treatment. J Am Coll Surg. 2008;207(1):13-9.

7. Coburn NG. Lymph nodes and gastric cancer. J Surg Oncol. 2009;99(4):199-206.

8. Colen K, Marcus S, Newman E, Berman R, Yee H, Hiotis S. Multiorgan resection for gastric cancer: intraoperative and computed tomography assessment of locally advanced disease is inaccurate. J Gastrointest Surg. 2004;8(7):897-900.

9. D'Amato A, Santella S, Cristaldi M, Gentili V, Pronio A, Montesani C. The role of extended total gastrectomy in advanced gastric cancer. Hepatogastroenterology. 2004;51(56):609-12.

10. Isozaki H, Tanaka N, Tanigawa N, Okajima K. Prognostic factors in patients with advanced gastric cancer with macroscopic invasion to adjacent organs treated with radical surgery. Gastric Cancer. 2000;3:202-10.

11. Jeong O, Choi W, Park Y. Appropriate selection of patients for combined organ resection in cases of gastric carcinoma invading adjacent organs. J Surg Oncol. 2009;100(2):115-20.

12. Kim J, Jang Y, Park S, Park S, Kim S, Mok Y, et al. Surgical outcomes and prognostic factors for T4 gastric cancers. Asian J Surg. 2009;32(4):198-204.

13. Kitamura K, Tani N, Koike H, Nishida S, Ichikawa D, Taniguchi $\mathrm{H}$, et al. Combined resection of the involved organs in $\mathrm{T} 4$ gastric cancer. Hepatogastroenterology. 2000;47(36):1769-72.

14. Lee J, Noh S, Lah K, Choi S, Min J. The prognosis of stage IV gastric carcinoma patients after curative resection. Hepatogastroenterology. 2001;48(42):1802-5.

15. Oñate-Ocaña L, Becker $\mathrm{M}$, Carrillo $\mathrm{J}$, Aiello Crocifoglio $\mathrm{V}$, Gallardo Rincón D, Brom Valladares R, et al. Selection of best candidates for multiorgan resection among patients with T4 gastric carcinoma. J Surg Oncol. 2008;98(5):336-42.

16. Otsuji E, Sawai K, Yamaguchi T, Hagiwara A, Okamoto K, Kobayashi S, et al. Results of combined complete removal of directly invaded organs with the stomach in patients with advanced gastric cancer. Hepatogastroenterology. 2002;49(48):1731-3.

17. Ozer I, Bostanci E, Orug T, Ozogul Y, Ulas M, Ercan M, et al. Surgical outcomes and survival after multiorgan resection for locally advanced gastric cancer. Am J Surg. 2009;198(1):25-30.

18. Saito H, Tsujitani S, Maeda Y, Fukuda K, Yamaguchi K, Ikeguchi $\mathrm{M}$, et al. Combined resection of invaded organs in patients with T4 gastric carcinoma. Gastric Cancer. 2001;4(4): 206-11.

19. Shchepotin I, Chorny V, Nauta R, Shabahang M, Buras R, Evans S. Extended surgical resection in T4 gastric cancer. Am J Surg. 1998;175(2):123-6.

20. Yong W, Wong W, Chan H, Soo K. Extended resection of locally advanced (T4) stomach cancer. Ann Acad Med Singapore. 2000;29(6):723.

21. American Joint Committee on Cancer. AJCC Cancer Staging Manual. 6th ed. Chicago: Springer; 2002.

22. Kwee R, Kwee T. Imaging in local staging of gastric cancer: a systematic review. J Clin Oncol. 2007;25(15):2107. 